\title{
MEMS enabled control of light-sheet microscopy optical beam paths
}

\author{
Spyridon Bakas ${ }^{1}$, Deepak Uttamchandani ${ }^{1}$, Hiroshi Toshiyoshi ${ }^{2}$, and Ralf Bauer ${ }^{1}$ \\ ${ }^{1}$ University of Strathclyde, Glasgow, United Kingdom \\ ${ }^{2}$ University of Tokyo, Tokyo, Japan \\ spyridon.bakas@strath.ac.uk
}

\begin{abstract}
The excitation and imaging optical beam paths for the design of a digitally controllable light-sheet microscopy system using a piezoelectric MEMS scanner and a thermal bimorph varifocal MEMS mirror, respectively, are presented.
\end{abstract}

Keywords-MEMS, micromirror, piezoelectric, electrothermal, biomedical imaging, light-sheet microscopy

\section{INTRODUCTION}

Fast 3D imaging of biomedical samples with cell scale resolution and low light doses is a distinct advantage of lightsheet fluorescence microscopy (LSM) systems [1]. The high cost per system is however still a major factor, with approaches like OpenSPIM [2] or the development of an adapter for a standard inverted microscope [3] aiming to mitigate this. The approach of using optical MEMS as active elements for miniaturisation and cost reduction was previously shown [4], with MEMS having the possibility to allow control of both the fluorescence excitation and imaging. The realization and control of a light-sheet can be achieved through a fast MEMS scanner and static positioning MEMS micromirror, similar to requirements from projection systems. Additionally, adding digital control of the imaging path for synchronisation can be enabled through electrical tuneable lenses or MEMS with focal length control [5], [6]. We will present the design and use of two MEMS micromirrors for control of both beam paths (excitation and imaging), together with the design and characterisation of the micromirrors in an LSM setup.

\section{MEMS DESIGN AND CHARACTERISTICS}

\section{A. Piezoelectric 2D scanning MEMS}

Full control of the light-sheet excitation beam path is through a silicon-on-insulator (SOI) MEMS micromirror with 2-axis control. Tilt actuation for both axes is achieved through piezoelectric PZT-layers deposited on "outside" static serpentine actuators and an "inside" resonant ring actuator. The design of the mirror is shown in Fig. 1(a), with a $\varnothing 1.1 \mathrm{~mm}$ mirror surface, coated with a thin Al layer. The micromirror, was fabricated by Stanley Electric Co. Inc. , and has a $100 \mu \mathrm{m}$ thick silicon device layer, a $2 \mu \mathrm{m}$ thick PZT actuation layer and a measured surface flatness of $<\lambda / 20$.

The resonant axis was tested using a Rigol DG1022Z signal generator and a $\times 20$ amplifier, with sinewave actuation using positive only signals with $10 \mathrm{~V}_{\mathrm{pp}}, 15 \mathrm{~V}_{\mathrm{pp}}$ and $20 \mathrm{~V}_{\mathrm{pp}}$ magnitude. The first tilt resonance of the inner actuation was detected at around $37.35 \mathrm{kHz}$, with total optical scan angles (TOSA) of

This work was supported by the Royal Academy of Engineering under the

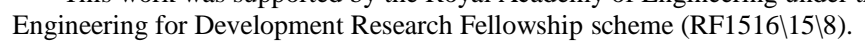

$9.2^{\circ}, 13.5^{\circ}$ and $18.0^{\circ}$, respectively, and a minimal spring softening behaviour (see Fig. 2(a)). The static tilt actuation was tested using a $40 \mathrm{~V}$ amplifier with an Arduino microcontroller for analogue control. A positive only voltage actuation was again used, with two piezoelectric actuator groups allowing bidirectional tilt [7]. The tilt response to actuation voltages up to $20 \mathrm{~V}$ is shown in Fig. 2(b), with a linear optical response of up to $\pm 3.3^{\circ}$.

\section{B. Thermal bimorph varifocal MEMS}

To allow digital control of the focal position of the imaging

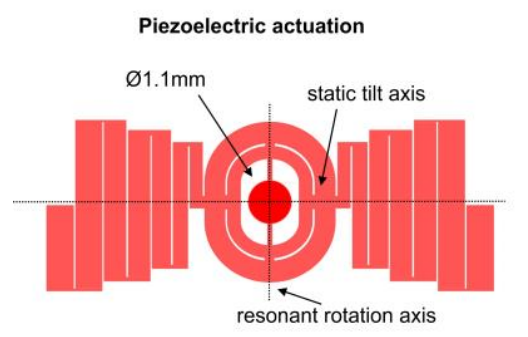

(a)

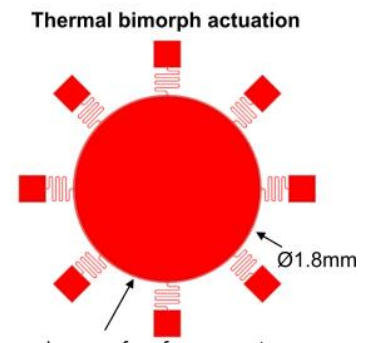

(b)

Fig. 1. Schematic of MEMS micromirrors for (a) light-sheet control using a 2D piezoelectric MEMS and (b) imaging control using a thermal bimorph MEMS
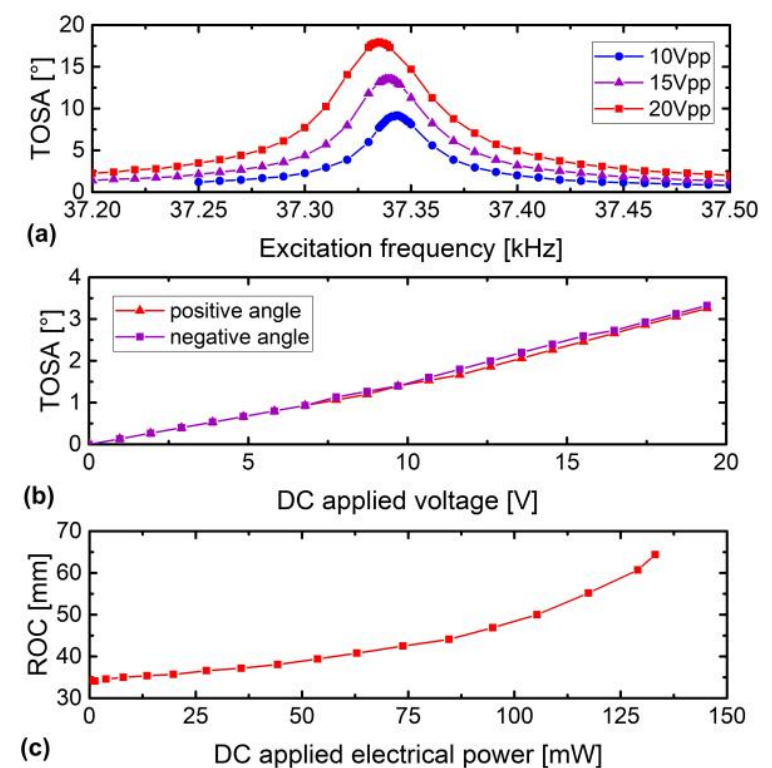

(c)

Fig. 2. MEMS optical characteristics: (a) Optical scan angle for resonant tilt of the piezoelectric MEMS (b) Optical scan angle for static tilt of the piezoelectric MEMS and (c) surface radius of curvature for the thermal bimorph MEMS. 


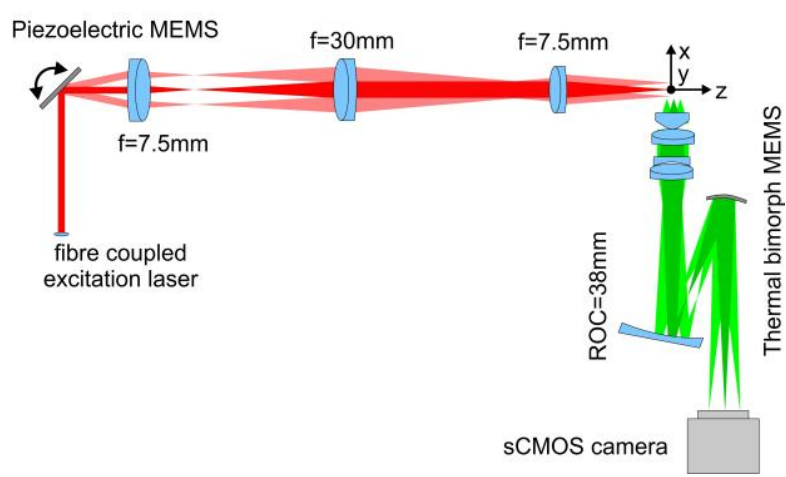

Fig. 3. Optical setup of light-sheet beam paths for excitation and imaging.

path, a varifocal MEMS micromirror with thermal bimorph actuation is used. The MEMS, fabricated by Memscap Inc. using their SOIMUMPs process, has a $1.8 \mathrm{~mm}$ mirror diameter attached to the substrate by 8 serpentine springs (see Fig. 1(b)). The $10 \mu \mathrm{m}$ thick silicon device layer has a $500 \mathrm{~nm}$ thick gold coating to enable thermal bimorph actuation.

The focal length change of the mirror surface was tested using a Veeco NT1100 surface profiler, with the initial radius of curvature (ROC) at room temperature being $33 \mathrm{~mm}$. Electrical actuation of the MEMS was through an additional channel of the Arduino amplifier with a maximum electrical power consumption of $130 \mathrm{~mW}$. The change of ROC is shown in Fig. 2(c), with a maximum curvature of $63 \mathrm{~mm}$ and a quadratic dependency of curvature on actuation power.

\section{OPTICAL DESIGN}

The optical schematic for both the excitation and imaging beam path of the designed LSM are shown in Fig. 3, using offthe-shelf optical components from Thorlabs Inc. The excitation path aims to produce a scanned laser light-sheet excitation with a laser beam spot size below $2 \mu \mathrm{m}$. It consists of a $488 \mathrm{~nm}$ Argon-Ion laser source (Omnichrome 532-AP-AO1) collimated onto a 2-axis PZT MEMS scanner at $45^{\circ}$ incidence, whose resonant scan creates the light-sheet, while the static axis allows its positioning relative to the imaging focal plane. The optical path is completed with a group of three lenses. A $\mathrm{f}=7.5 \mathrm{~mm}$ lens is positioned $5 \mathrm{~mm}$ from the mirror, followed by a $36.4 \mathrm{~mm}$ spaced $f=30 \mathrm{~mm}$ lens and a final $f=7.5 \mathrm{~mm}$ focus lens positioned at a $34 \mathrm{~mm}$ distance. The excitation path is designed to produce a focal plane with $5.2 \mathrm{~mm}$ working distance after the last lens.

The orthogonal imaging path was designed with a target resolution below $1 \mu \mathrm{m}$ and consists of two lens groups with $4.5 \mathrm{~mm}$ spacing, a silver mirror with $38 \mathrm{~mm}$ ROC and the

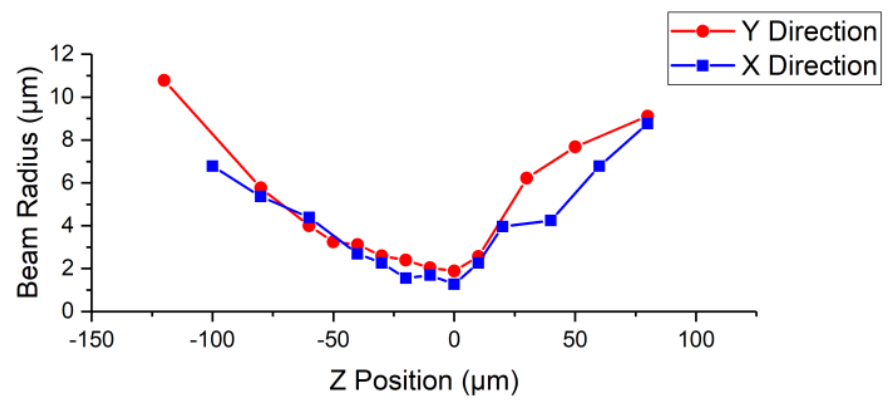

Fig. 4. Light-sheet excitation beam propagation characteristics in the focal plane area for major beam axes.

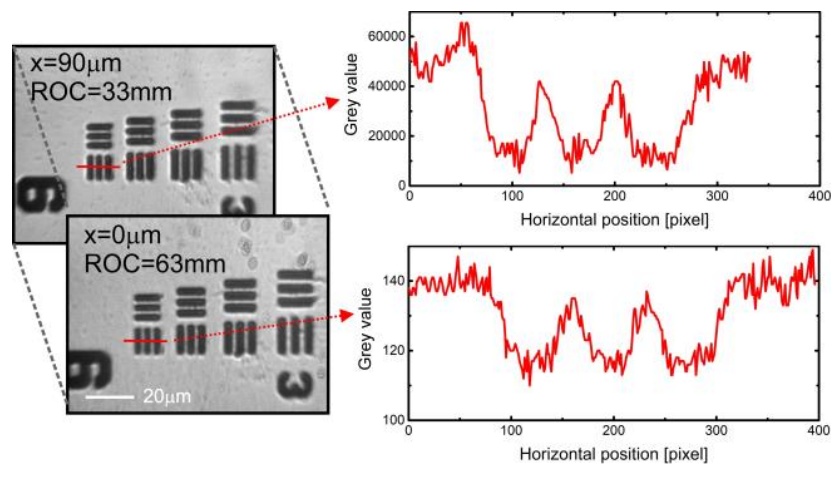

Fig. 5. Imaging beam path characteristics at maximum $\mathrm{x}$-positions with 1951 USAF resolution target group 7-6 cross-sections.

thermal bimorph MEMS which focuses the image planes onto the CMOS camera (FLIR Grasshopper 3). The design was simulated using Optenso's Optalix software, showing a field of view (FOV) of $114 \mu \mathrm{m}$ by $84 \mu \mathrm{m}$, with a focal plane shift of $110 \mu \mathrm{m}$ over the addressable range of the varifocal mirror.

\section{RESULTS}

The evaluation of the excitation path was achieved performing a knife edge test in the focal plane area of the laser propagation using a motorized stage (Thorlabs MTS25-Z8) in order to achieve measurement steps below $1 \mu \mathrm{m}$. The edge response was captured with the use of an optical power meter (Thorlabs PM100A). The measured beam radius through the focal position is presented in Fig. 4 and shows a FOV of $100 \mu \mathrm{m}$ where the excitation beam radius remains below $4 \mu \mathrm{m}$, with a centre focal spot radius of around $1.5 \mu \mathrm{m}$. In this area the light-sheet height is around $550 \mu \mathrm{m}$, whereas the width is considered equal to the minimum focal spot diameter.

The imaging path was evaluated using a 1951 USAF resolution target (Thorlabs R1DS1P), mounted on a precision translation stage and using a backlit illumination. The resolution target was manually positioned through the focal range, with the varifocal mirror optimised at each position for best image sharpness. The resulting image performance, shown in Fig. 5, has an addressable depth range of $90 \mu \mathrm{m}$ with an image FOV of $105 \mu \mathrm{m}$ by $78 \mu \mathrm{m}$. The resolution group 7-6 is still clearly identifiable, which shows an image resolution well below $2.2 \mu \mathrm{m}$.

\section{REFERENCES}

[1] R. M. Power and J. Huisken, "A guide to light-sheet fluorescence microscopy for multiscale imaging - supplimentary notes," Nat. Methods, vol. 14, no. 4, pp. 360-373, 2017.

[2] P. G. Pitrone et al., "OpenSPIM: An open-access light-sheet microscopy platform," Nat. Methods, vol. 10, no. 7, pp. 598-599, 2013.

[3] Z. Guan et al., "Compact plane illumination plugin device to enable light sheet fluorescence imaging of multi-cellular organisms on an inverted wide-field microscope," Biomed. Opt. Express, vol. 7, no. 1, pp. 194 208, 2016.

[4] R. Bauer and D. Uttamchandani, "MEMS micromirror based light sheet generator for biomedical imaging," in 2017 International Conference on Optical MEMS and Nanophotonics (OMN), 2017, pp. 1-2.

[5] A. Paterson et al. "Range Extension of a Bimorph Varifocal Micromirror Through Actuation by a Peltier Element," IEEE J. Sel. Top. Quantum Electron., vol. 21, no. 4, p. 2701007, 2015.

[6] T. Liu and D. L. Dickensheets, "MEMS 3-dimensional scanner for handheld confocal microscope," in 2017 International Conference on Optical MEMS and Nanophotonics (OMN), 2017, pp. 1-2.

[7] S. Jeon and H. Toshiyoshi, "MEMS tracking mirror system for a bidirectional free-space optical link," Appl. Opt., vol. 56, no. 24, pp. 6720-6727, 2017. 